\title{
Phase-Resolved UV Spectroscopy of V834 Cen and MR Ser in High and Intermediate States
}

\author{
Magdy R. Sanad ${ }^{1}$, Matthew Bobrowsky ${ }^{2}$ \\ ${ }^{1}$ Astronomy Department, National Research Institute of Astronomy and Geophysics, Helwan, Cairo, Egypt \\ ${ }^{2}$ Department of Physics, University of Maryland, College Park, USA \\ Email:mrsanad1@yahoo.com,msb@umd.edu
}

Received August 12, 2012; revised September 15, 2012; accepted September 23, 2012

\begin{abstract}
We present a spectroscopic study of two polar systems, V834 Cen and MR Ser observed with IUE (International Ultraviolet Explorer) obtained during the period 1982-1991 and 1982-1987 for both systems respectively, to diagnose the ultraviolet fluxes of C IV $1550 \AA$ and He II $1640 \AA$ emission lines originating in the accretion stream during different orbital phases. Two spectra for both systems showing the variations in line fluxes at different orbital phases in high and intermediate states are presented. We concentrated on calculating the line fluxes of C IV \& He II emission lines. Our results show that there is spectral variability for the line fluxes at different times, similar to that for optical spectrum of V834 Cen [1] and similar to that for the light curves of MR Ser [2]. We attribute this spectral variability to the variations of mass accretion rate [3]. Also we found that the line fluxes of both CIV and He II for V834 Cen are greater than the line fluxes of MR Ser.
\end{abstract}

Keywords: Accretion-Binaries; Close-Stars; Individual; V834 Cen-MR Ser; Ultraviolet Stars; Magnetic Fields-White Dwarfs

\section{Introduction}

Polars are interacting binary stars. The primary is a white dwarf with a magnetic field of the order of a few tens of MegaGauss. The secondary is a lower Main-Sequence red dwarf which fills its Roche Lobe. It loses gas at the inner Lagrangian point and this falls towards the primary in a stream. The gas in the stream is ionized by collisions and X-rays from the accretion region on the primary, so at some point in the stream's trajectory the magnetic energy density of the primary's field is sufficient to divert the stream from its free fall trajectory and force it to follow the field lines. Accretion, therefore, occurs over a small area near one or both of the magnetic poles of the white dwarf. Material in the stream is travelling at roughly the escape velocity of the white dwarf when it approaches the surface of the white dwarf, so a strong shock forms in the accretion flow close to the surface. Being interacting red dwarf-white dwarf pairs, polars are a class of Cataclysmic variable (CV). For a review of cataclysmic variables and polars, see [4-7].

V834 Cen is a well-studied polar with an orbital period $P_{\text {orb }}=101.5$ minutes and the high- $(V \approx 14)$ and low$(V \approx 17)$ luminosity states typical of this class of binaries. The magnetic nature of the white dwarf is demonstrated directly by the linear and circular polarization present in its high states $[3,8,9]$ and the Zeeman absorption features and cyclotron emission features present in its low states [10-13], from which a magnetic field strength of $23 \mathrm{MG}$ is inferred.

Reference [14] find $d \approx 77 \mathrm{pc}$, based on a detection of the secondary in a near-IR spectrum; reference [15] find $M_{w d}=0.54-0.64 \mathrm{M}_{\odot}$, based on fits of a Ginga X-ray spectrum; reference [16] finds $M_{w d}=0.64-0.68 \mathrm{M}_{\odot}$, based on fits of an RXTE X-ray spectrum; and [1] use phase-resolved optical spectra to determine $M_{w d} \approx 0.66$ $\mathrm{M}_{\odot}$. Reference [17] advocates $i=450 \pm 90, \beta=250 \pm 50$, and $\psi=400 \pm 50$, based on values compiled from the literature.

Reference [18] observed $P$ Cygni-like profiles in the emission lines of CIV $1550 \AA$ and He II $1640 \AA$ in an International Ultraviolet Explorer (IUE) spectrum of V834 Cen. They suggested that such profiles might be due to a magnetically confined outflowing wind in the system. They obtained a blackbody temperature from their IUE spectra of about $25,500 \mathrm{~K}$ when the system was in an intermediate state.

Reference [19] made three later IUE observations of V834 Cen. In two of these observations, the system was in a high-luminosity state, whilst in the other it was observed in its low state. Their spectra showed no evidence of $P$ Cygni profiles in the lines in either state. 
During the star's low state the observed ultraviolet continuum flux distribution was found to be consistent with the blackbody emission expected from a white dwarf with a temperature of about $26,500 \mathrm{~K}$. They found that, a residual steep component was present below $2000 \AA$. This residual component, they suggested, could be explained in terms of a heated region, probably associated with the dominant accreting polar cap.

Reference [20] showed dips in the low-energy X-ray flux occurring over a short phase interval which includes the time of optical minimum. They also observed a hard medium - energy spectral component, the light curve of which peaks after the time of optical minimum.

MR Ser is a well-studied polar with an orbital period $P_{\text {orb }}=113.56$ minutes [21]. Reference [1] by using the method of [22] and a slightly revised $S_{k}$ vs. (V-K) -relation based on more recent observations of single late type M-dwarfs $[23,24]$ determined the distance to MR Ser to $d$ $=139 \pm 13$ pc. $[21,25-27]$ find $M_{w d} \approx 0.62-0.67 \mathrm{M}_{\odot}$ with an inclination of $i=45 \pm 50$.

The magnetic nature of the white dwarf is demonstrated by Zeeman features seen against the bright cyclotron background in its high accretion states and the Zeeman absorption lines and cyclotron emission lines present in its low accretion states [21] from which a magnetic field strength of $25 \mathrm{MG}$ is inferred. K-band spectroscopy shows a M8 V secondary with normal carbon abundances [28]. Reference [25] obtained a temperature of the white dwarf to be $14,200 \mathrm{~K}$ based on the unambiguous signature of the white dwarf in the FUV.

References [29,30] studied MR Ser in the UV by using IUE observations, they found that, MR Ser has a steeper UV distribution, with a possibility for a hot RayleighJeans component at wavelengths less than $1600 \AA$. Also they found that, during intermediate states of MR Ser, there is signature of decreased line emission and narrower line widths as compared to the high states.

Spectral observations and data reductions are presented in Section 2. The results and the discussions are presented in Section 3 and its implications concerning the spectral behavior of both V834 Cen and MR Ser systems and the reasons behind this variability. In Section 4, a comparison between V834 Cen and MR Ser is made. Finally, concluding remarks about this work are presented in Section 5.

\section{Observations and Data Reductions: IUE SWP Low Resolution Spectra of V834 Cen and MR Ser in High State}

The IUE low resolution short wavelength spectra have been retrieved from the INES (IUE Newly Extracted Spectra) system through its principle centre at http://ines.vilspa.esa.es. A Full description of the INES system for low resolution data is given in $[31,32]$. IUE archive contains 20 observations for V834 Cen and 9 observations for MR Ser between high and intermediate states. In the absence of an accretion disk, the high and low states of polars are likely to be consequences of changes in the mass-transfer rate in the system. It has been argued that the high/low states of polars are related to the atmospheric magnetic activity of the mass-donor star. In the star spot model [33], a low state is caused by a temporary cessation of mass transfer, due to a magnetic spot on surface of the Roche-lobe filling mass-donor star traversing the inner Lagrange (L1) point [34].

All spectra were taken in the low resolution mode of IUE and through the large aperture, leading to a spectral resolution of $\sim 6 \AA$ [35]. We found that there are $18 \mathrm{ob}-$ servations in high and intermediate states for V834 Cen, and 9 observations in high and intermediate states for MR Ser (Table 1). The data were analyzed by the MIDAS software. We referenced the spectra to the orbital phase of the V834 Cen using the ephemeris of [21], and of MR Ser using the ephemeris of [21].

The spectra were inspected individually in the 1150 $1950 \AA$ region to identify and reject noisy and overexposed or underexposed data. The most suitable emission lines for flux measurements are C IV $1550 \AA$ and He II $1640 \AA$. Representative examples of emission lines are given in Figures 1-4, showing the variations of line fluxes and line widths at different times. These lines arise from the accretion stream.

\section{Results and Discussions}

\subsection{Method of Calculating the Line Fluxes of C IV \& He II Emission Lines}

Our method of calculating the integrated fluxes of emission lines depends on using Gaussian function as follows: It is based on fitting the observed portions of the emission line wings with a Gaussian function. We used the integrate/line command in the ESO/MIDAS suite to interactively determine the continuum level and integrate the flux of the emission lines above this continuum. For the calculations of line fluxes, we calculated the integrated fluxes for these emission lines $\left(\mathrm{erg} \mathrm{cm}^{-2} \cdot \mathrm{s}^{-1}\right)$.

\subsection{Spectral Behavior of C IV \& He II Emission Lines for V 834 Cen}

The C IV emission line at $1550 \AA$ is resonance, high excitation emission line, while He II emission line at $1640 \AA$

Table 1. IUE data sets for V834 Cen and MR Ser.

\begin{tabular}{ccccc}
\hline System & Dispersion & Aperture & $\begin{array}{c}\text { Period } \\
\text { (minute) }\end{array}$ & $\begin{array}{c}\text { Number of } \\
\text { IUE spectra }\end{array}$ \\
\hline V834 Cen & Low & Large & 101.5 & 18 \\
MR Ser & Low & Large & 113.5 & 9 \\
\hline
\end{tabular}




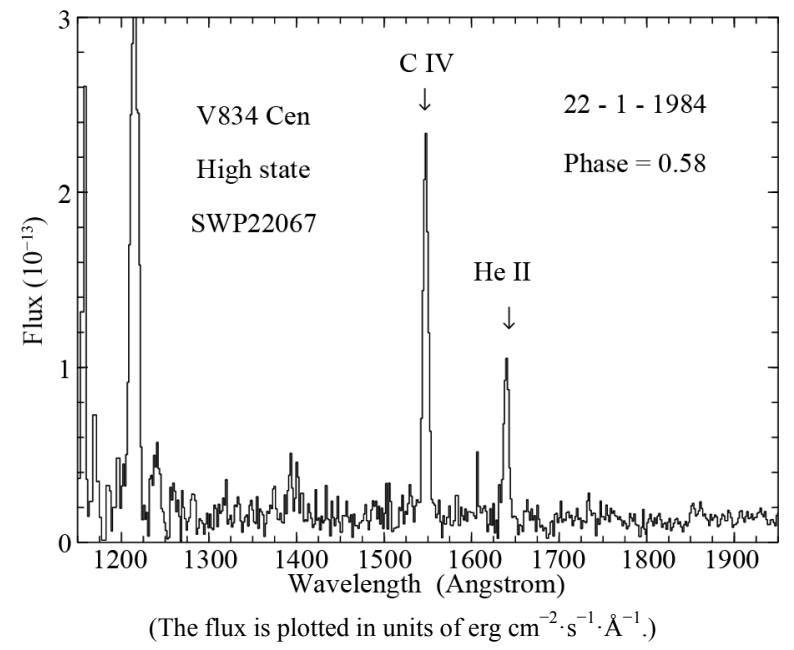

Figure 1. IUE spectrum of V834 Cen in high state at phase 0.58

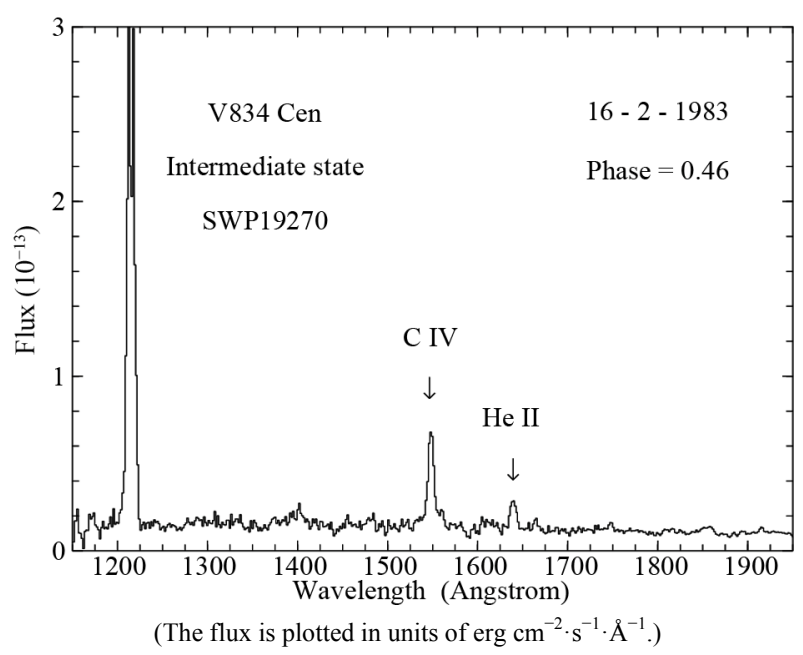

Figure 2. IUE spectrum of V834 Cen in intermediate state at phase 0.46 .

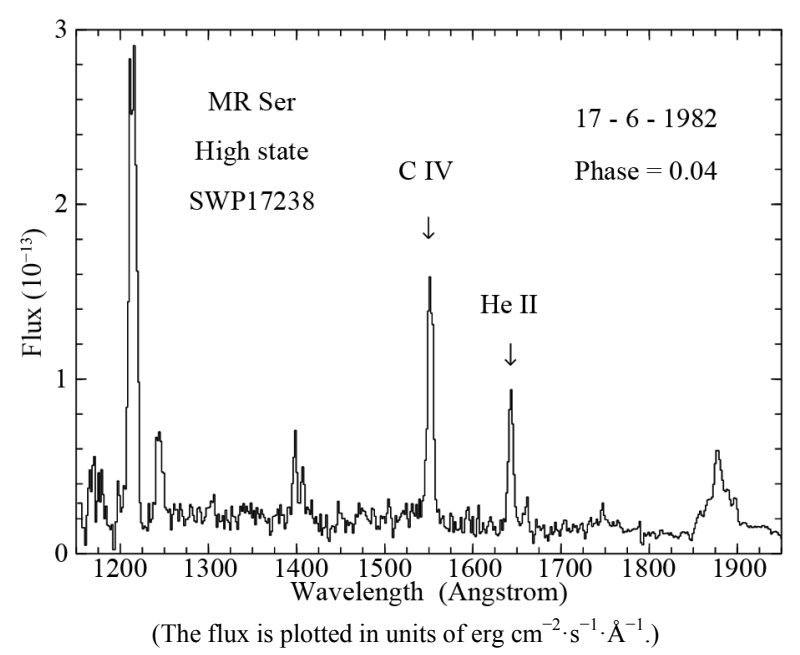

Figure 3. IUE spectrum of MR Ser in high state at phase 0.04 .

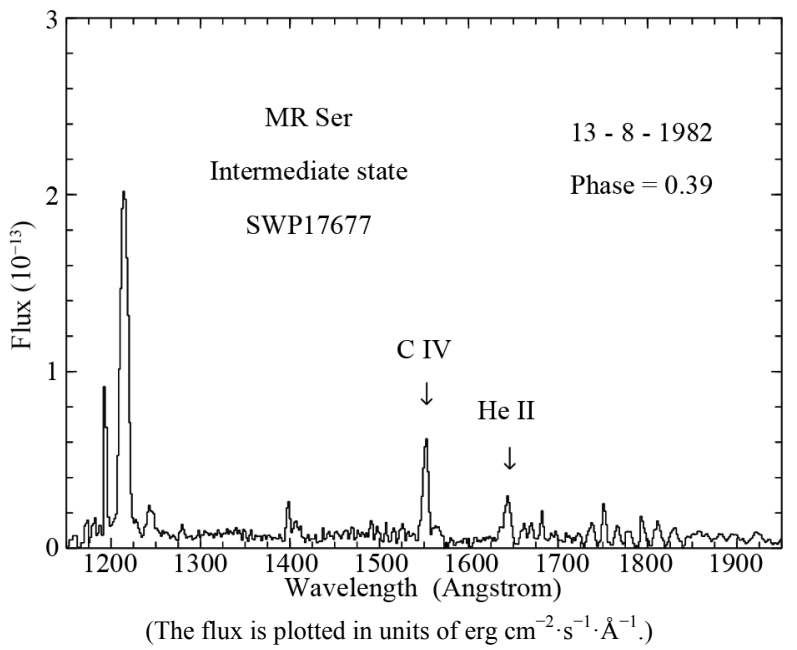

Figure 4. IUE spectrum of MR Ser in intermediate state at phase 0.39 .

is recombination line. In the low state the ultraviolet spectrum of V834 Cen can be interpreted as the emission from a hot white dwarf with $\mathrm{T}=26,500 \mathrm{~K}$, and the $\mathrm{R}$ and I magnitudes can be accounted for by a Roche lobe filling secondary of spectral type between M3 V and M8 V at a distance between 50 and 200 pc. In the high state, the UV continuum is described by a power law $F_{\lambda} \alpha \lambda^{-\alpha}$ with $\alpha=1.9$, while the optical and Infrared magnitudes lie above the extrapolation of this power low [19].

The continuum flux distribution rises steeply at short wavelength and shows the effects of interstellar extinction. Best fit parameters for a blackbody spectrum are $\mathrm{T}$ $=25,500 \pm 2300 \mathrm{~K}[18]$.

There are complex phase-dependent emission line profiles, which are clearly resolved into at least four components. A narrow spectral feature is interpreted as being produced in a region close to the secondary. The relative phasing, velocity amplitudes and widths of the remaining spectral components indicate that they are formed in parts of the accretion stream closer to the white dwarf and imply considerable structure within the accretion column [36].

There are changes in the accretion geometry caused by an increase in the mass accretion rate and/or the clumpiness of the flow. The EUVE spectra are well described by either a blackbody or a pure $\mathrm{H}$ stellar atmosphere absorbed by a neutral hydrogen column density [3].

Figures 5 and 6 represent the behavior of line fluxes with orbital phases for C IV \& He II emission lines. The line fluxes are vary at different orbital phases between high, intermediate and low values, the reasons for this behavior are as follows:

The C IV \& He II emission lines are expected to be formed in the accretion stream, where the mass accretion rate is variable [3] leading to variations of both density 


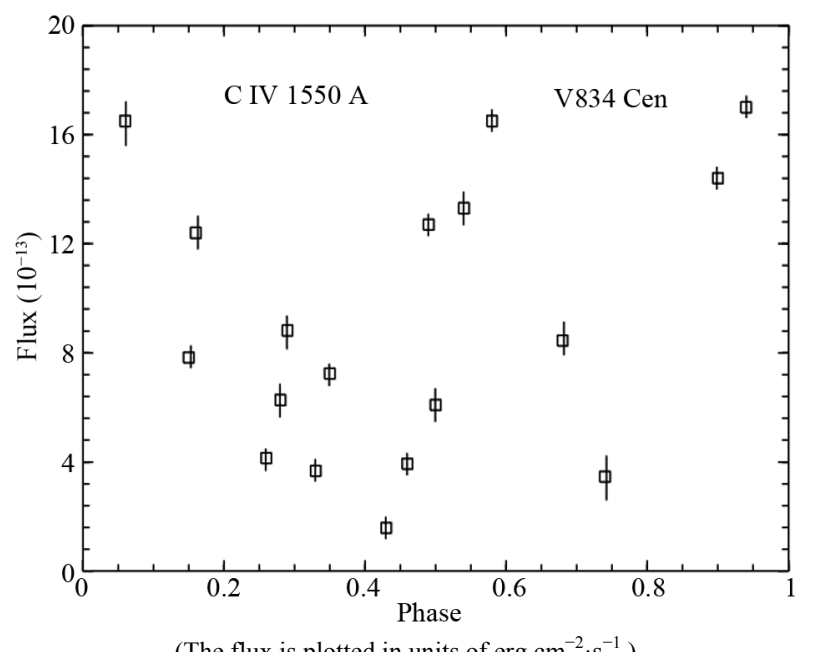

(The flux is plotted in units of $\mathrm{erg} \mathrm{cm}^{-2} \cdot \mathrm{s}^{-1}$.)

Figure 5. Spectral behavior of C IV line flux with orbital phase. The $1 \sigma$ error bars are shown on each data point.

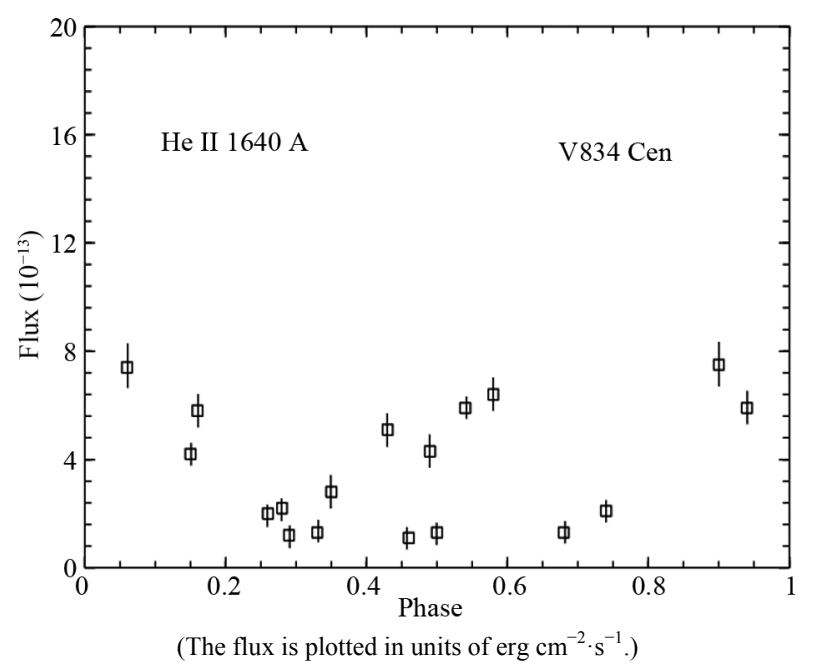

Figure 6. Spectral behavior of He II line flux with orbital phase. The $1 \sigma$ error bars are shown on each data point.

and temperature, this in turn affect the values of fluxes of C IV \& He II emission lines. Figures 5 and $\mathbf{6}$ reveal that there are high values of fluxes around orbital phases 0 , $0.10,0.60$, and 0.90 , intermediate values at around orbital phases 0.30 and 0.70 , and low values at around orbital phases 0.40 , and 0.8 , similar to the variations of optical spectra for this system [21]. The errors for measured line fluxes is in the range of $1 \sigma$. The uncertainty in the values of line fluxes was determined using the procedures outlined by $[37,38]$.

\subsection{Spectral Behavior of C IV \& He II Emission Lines for MR Ser}

The C IV resonance emission line at $1550 \AA$ and He II recombination line at $1640 \AA$ for MR Ser behave approximately as the C IV \& He II for V834 Cen. Figures
7 and $\mathbf{8}$ represent the behavior of line fluxes with phase for C IV \& He II of MR Ser. In the low state the ultraviolet emission lines are approximately absent [1] while in the intermediate and high states the ultraviolet emission lines are appear and originating in the accretion stream $[12,28,33]$.

The spectral variations of line fluxes of C IV \& He II emission lines of MR Ser in Figures $\mathbf{7}$ and $\mathbf{8}$ are variable at different phases between high, intermediate, and low values, the reasons are similar to that interpreted for the spectral behavior of C IV \& He II emission lines of V834 Cen, depending on the variations of density and temperature of accretion stream as a result of changing the rate of mass loss from the secondary star to the white dwarf. The errors for measured line fluxes are in the range of $1 \sigma$. The uncertainty in the values of line fluxes was determined using the procedures outlined by $[6,16]$.

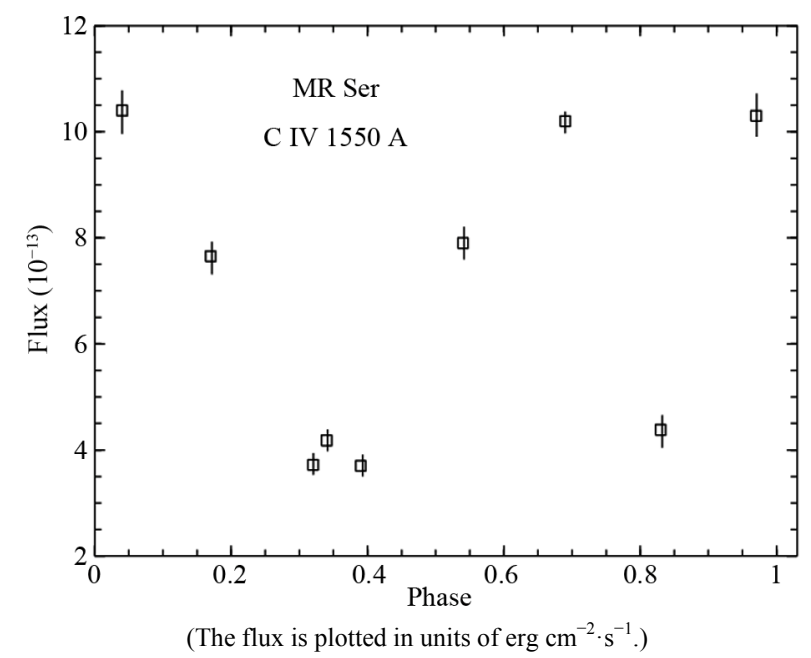

Figure 7. Spectral behavior of C IV line flux with orbital phase. The $1 \sigma$ error bars are shown on each data point.

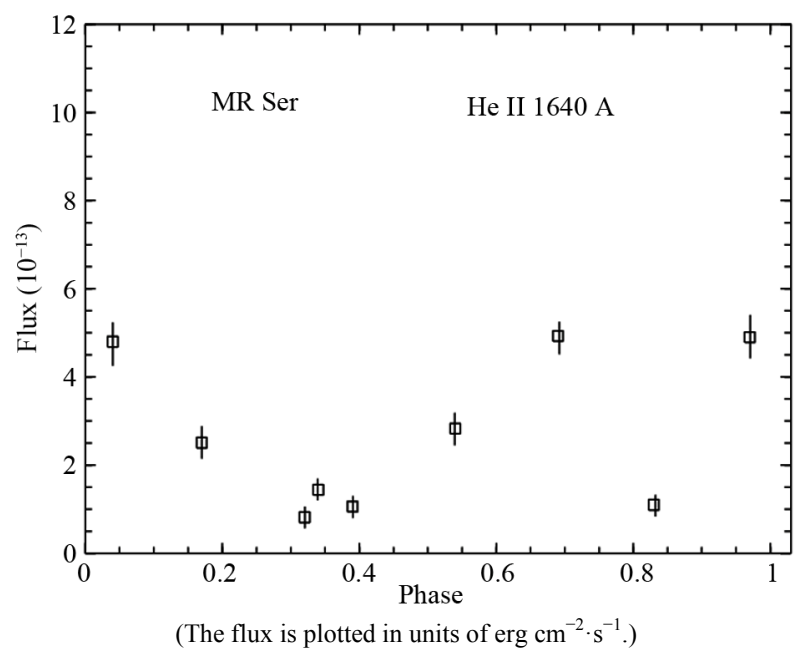

Figure 8. Spectral behavior of He II line flux with orbital phase. The $1 \sigma$ error bars are shown on each data point. 


\section{Comparison between the Spectral Behavior of V834 Cen and MR Ser in Intermediate and High States}

Figures 5 and 6 describe the spectral behavior of V834 Cen in the form of relations between line fluxes with orbital phase for C IV \& He II emission lines, and their corresponding Figures $\mathbf{7}$ and $\mathbf{8}$ for MR Ser. We found the following:

1) The spectral behavior of C IV \& He II emission lines for both V834 Cen and MR Ser in the form of relations between line fluxes with orbital phases is approximately the same, showing high values of line fluxes at some orbital phases, intermediate values at another orbital phases and low values at another orbital phases.

2) The line fluxes of C IV \& He II emission lines of V834 Cen are slightly greater than the line fluxes of C IV \& He II emission lines of MR Ser as appeared in Figures $\mathbf{5}$ and $\mathbf{7}$ and Tables $\mathbf{2}$ and $\mathbf{3}$.

It is known that the characteristics of the white dwarf will determine to a large extent the physics involved in the accretion process, thereby the form of the emitted spectrum and the accretion process will modify the white dwarf characteristics, the accreted matter will enrich the white dwarf with heavy elements. The difference in mass between the two white dwarfs for two systems is small, while the distance to V834 Cen is smaller than the distance to MR Ser, this interpreting the small difference of line fluxes between two systems.

\section{Conclusions}

We have presented low-resolution spectral observations of the two AM Herculis binaries V834 Cen and MR Ser during intermediate and high accretion states. These observations show emission lines originating in the accretion stream. Our analysis of the phase dependent emission lines suggests that is changing with phase between intermediate and high accretion states. We found the following results:

1) Only in intermediate and high accretion states of V834 Cen and MR Ser, do spectral emission lines in the accretion stream appear.

2) There is spectral variability in the form of relations between line fluxes with orbital phase of V834 Cen and MR Ser at different times as a result of changing the mass accretion rate.

3) The spectral behavior of C IV \& He II emission lines for both V834 Cen and MR Ser is approximately the same, showing high values of the line fluxes at some orbital phases, intermediate values at other orbital phases, and low values at other orbital phases.

4) The line fluxes of V834 Cen is slightly greater than the line fluxes of MR Ser.
Table 2. Line fluxes of C IV \& He II of V834 Cen in units of $\left(\mathrm{erg} \mathrm{cm} \mathrm{cm}^{-2} \cdot \mathrm{s}^{-1}\right)$.

\begin{tabular}{cccc}
\hline Image ID & $\begin{array}{c}\text { Line flux } \\
\text { of C IV }\end{array}$ & $\begin{array}{c}\text { Line flux } \\
\text { of He II }\end{array}$ & $\begin{array}{c}\text { Orbital } \\
\text { phase }\end{array}$ \\
\hline SWP15943 & $3.46 \mathrm{E}-013$ & $2.10 \mathrm{E}-013$ & 0.74 \\
SWP19269 & $3.67 \mathrm{E}-013$ & $1.38 \mathrm{E}-013$ & 0.33 \\
SWP19270 & $3.93 \mathrm{E}-013$ & $1.12 \mathrm{E}-013$ & 0.46 \\
SWP19818 & $6.09 \mathrm{E}-013$ & $1.37 \mathrm{E}-013$ & 0.50 \\
SWP19819 & $7.83 \mathrm{E}-013$ & $4.27 \mathrm{E}-013$ & 0.15 \\
SWP22066 & $1.65 \mathrm{E}-012$ & $7.46 \mathrm{E}-013$ & 0.06 \\
SWP22067 & $1.65 \mathrm{E}-012$ & $6.46 \mathrm{E}-013$ & 0.58 \\
SWP22068 & $8.82 \mathrm{E}-013$ & $1.21 \mathrm{E}-013$ & 0.29 \\
SWP22069 & $1.70 \mathrm{E}-012$ & $5.98 \mathrm{E}-013$ & 0.94 \\
SWP22070 & $1.33 \mathrm{E}-012$ & $5.98 \mathrm{E}-013$ & 0.54 \\
SWP22071 & $1.24 \mathrm{E}-012$ & $5.85 \mathrm{E}-013$ & 0.16 \\
SWP22072 & $1.44 \mathrm{E}-012$ & $7.51 \mathrm{E}-013$ & 0.90 \\
SWP22073 & $1.27 \mathrm{E}-012$ & $4.31 \mathrm{E}-013$ & 0.49 \\
SWP22570 & $4.14 \mathrm{E}-013$ & $2.03 \mathrm{E}-013$ & 0.26 \\
SWP22571 & $7.24 \mathrm{E}-013$ & $2.86 \mathrm{E}-013$ & 0.35 \\
SWP27857 & $8.45 \mathrm{E}-013$ & $1.32 \mathrm{E}-013$ & 0.68 \\
SWP27858 & $6.27 \mathrm{E}-013$ & $2.20 \mathrm{E}-013$ & 0.28 \\
SWP40570 & $1.58 \mathrm{E}-013$ & $5.13 \mathrm{E}-014$ & 0.43 \\
\hline & & &
\end{tabular}

Table 3. Line fluxes of C IV \& He II of MR Ser in units of $\left(\operatorname{erg~cm} \mathrm{cm}^{-2} \cdot \mathrm{s}^{-1}\right)$.

\begin{tabular}{cccc}
\hline Image ID & $\begin{array}{c}\text { Line flux } \\
\text { of CIV }\end{array}$ & $\begin{array}{c}\text { Line flux } \\
\text { of He II }\end{array}$ & $\begin{array}{c}\text { Orbital } \\
\text { phase }\end{array}$ \\
\hline SWP17238 & $1.04 \mathrm{E}-012$ & $4.80 \mathrm{E}-013$ & 0.04 \\
SWP17677 & $3.70 \mathrm{E}-013$ & $1.06 \mathrm{E}-013$ & 0.39 \\
SWP17714 & $7.56 \mathrm{E}-013$ & $2.51 \mathrm{E}-013$ & 0.17 \\
SWP19366 & $4.38 \mathrm{E}-013$ & $1.10 \mathrm{E}-013$ & 0.83 \\
SWP19367 & $1.03 \mathrm{E}-012$ & $4.90 \mathrm{E}-013$ & 0.97 \\
SWP19368 & $1.02 \mathrm{E}-012$ & $4.93 \mathrm{E}-013$ & 0.69 \\
SWP19369 & $7.90 \mathrm{E}-013$ & $2.83 \mathrm{E}-013$ & 0.54 \\
SWP20182 & $4.18 \mathrm{E}-013$ & $1.44 \mathrm{E}-013$ & 0.34 \\
SWP30312 & $3.72 \mathrm{E}-013$ & $8.24 \mathrm{E}-014$ & 0.32 \\
\hline
\end{tabular}

\section{Acknowledgements}

M.B. acknowledges support for this work from NASA, through grant number HST-AR-10304.04 from the Space Telescope Science Institute, which is operated by the Association of Universities for Research in Astronomy, Inc., under NASA contract NAS5-26555.

\section{REFERENCES}

[1] A. D. Schwope, H.-C. Thomas, K. Beuermann and K. Reinsch, "A Spectroscopic Ephemeris of the Secondary 
Star in the AM Herculis Binary V834 Centauri," Astronomy \& Astrophysics, Vol. 267, No. 1, 1993, p. 103.

[2] M. P. Diaz and D. Cieslinski, "Multiline Dopple Imaging Of MR Ser in High State," Astronomical Journal, Vol. 137, No. 1, 2009, p. 296.

[3] C. W. Mauche, "Extreme Ultraviolet Explorer Phase-Resolved Spectroscopy of V834 Centauri," The Astrophysical Journal, Vol. 578, No. 1 2002, p. 439. doi:10.1086/342364

[4] K. Beuermann, "Publications of the Astronomical Society of the Pacific," Publications of the Astronomical Society, Vol. 114, No. 794, 2002, p. 427. doi:10.1086/342494

[5] M. Cropper, "The Polars," Space Science Reviews, Vol. 54, 1990, p. 195.

[6] B. Warner, "Cataclysmic Variable Stars," Cambridge University Press, Cambridge, 1995.

[7] K. Wu, M. Cropper, G. Ramsay, C. J. Saxton and C. Bridge, "Accretion Flow in Magnetic Cataclysmic Variables," Chinese Journal of Astronomy and Astrophysics, Vol. 3, Suppl. 1, 2003, p. 235. doi:10.1088/1009-9271/3/S1/235

[8] M. Cropper, J. W. Menzies and S. Tapia, "E1405-451Three Seasons of Polarimetry and Photometry," Monthly Notices of the Royal Astronomical Society, Vol. 218, 1986, p. 201.

[9] M. Cropper, "The Accretion Region in AM HER Systems - Insights from polarimetry of V834 CEN," Monthly Notices of the Royal Astronomical Society, Vol. 236, 1989, p. 935.

[10] L. Ferrario, D. T. Wickramasinghe, J. Bailey, J. H. Hough and I. R. Tuohy, "Detection of Photospheric Zeeman Features and Cyclotron Emission Lines in V834 CEN in a Low State," Monthly Notices of the Royal Astronomical Society, Vol. 256, No. 2, 1992, p. 252.

[11] S. Tapia, "E1405-451 and E1013-477," International Astronomical Union Circulars, IAUC, Nos, 1982, pp. 3684-3685.

[12] N. Visvanathan and I. Tuohy, "Linear Polarization Measurements of the AM Herculis Objects H0139-68 and E1405-451," Astrophysical Journal, Vol. 275, 1983, pp. 709-712. doi:10.1086/161568

[13] A. D. Schwope and K. Beuermann, "Cyclotron and Zeeman Spectroscopy of V834 Centauri," Astronomy and Astrophysics, Vol. 238, No. 1-2, 1990, p. 173.

[14] E. M. Puchnarewicz, K. O. Mason, P. G. Murdin and D. T. Wickramasinghe, "Low-State Spectroscopy of V834 CEN (E1405-451)," Monthly Notices of the Royal Astronomical Society, Vol. 244, 1990, p. 20.

[15] M. Cropper, K. Wu, G. Ramsay and A. Kocabiyik, "Effects of Gravity on the Structure of Post-Shock Accretion Flows in Magnetic Cataclysmic Variables," Monthly Notices of the Royal Astronomical Society, Vol. 306, No. 3, 1999, pp. 684-690. doi:10.1046/j.1365-8711.1999.02570.x

[16] G. Ramsay, "Determining the Mass of the Accreting White Dwarf in Magnetic Cataclysmic Variables Using RXTE Data," Monthly Notices of the Royal Astronomical
Society, Vol. 314, No. 2, 2000, pp. 403-408 doi:10.1046/j.1365-8711.2000.03239.x

[17] M. Cropper, "The Orientations of the Magnetic Pole in AM HER Systems-Implications for Synchronous Rotation and Selection Effects," Monthly Notices of the Royal Astronomical Society, Vol. 231, 1988, p. 597.

[18] J. A. Nousek and S. H. Pravdo, "IUE Observations of E1405-451-A New AM Herculis Type Cataclysmic Variable," Astrophysical Journal, Vol. 266, 1983, pp. L39-L43. doi:10.1086/183974

[19] L. Maraschi, A. Treves, E. G. Tanzi, M. Mouchet, A. Lauberts, C. Motch, J. M. Bonnet Bidaud and M. M. Phillips, "Coordinated UV and Optical Observations of the AM Herculis Object E1405-451 in the High and Low States," Astrophysical Journal, Vol. 285, 1984, pp. 214222. doi:10.1086/162494

[20] J. P. Osborne, et al., "X-Ray Astronomy 84: International Symposium on X-Ray Astronomy, Bologna," Institute of Space and Astronautical Science, 1984, p. 59.

[21] A. D. Schwope, K. Beuermann, S. Jordan and H. C. Thomas, "Cyclotron and Zeeman Spectroscopy of MR Serpentis in Low and High States of Accretion," Astronomy and Astrophysics, Vol. 278, No. 2, 1993, p. 487.

[22] J. Bailey, "The Distances of Cataclysmic Variables," Monthly Notices of the Royal Astronomical Society, Vol. 197, 1981, p. 31.

[23] G. Berriman and N. Reid, "Observations of M Dwarfs beyond 2.2 Microns," Monthly Notices of the Royal Astronomical Society, Vol. 227, 1987, p. 315.

[24] N. Reid and G. Gilmore, "New Light on Faint Stars. VInfrared Photometry and the H-R Diagram for Very Low Mass Dwarfs," Monthly Notices of the Royal Astronomical Society, Vol. 206, 1984, p. 19.

[25] S. Araujo-Betancor, B. T. Gänsicke, K. S. Long, K. Beuermann, D. de Martino, E. M. Sion and P. Szkody, "Far-Ultraviolet Spectroscopy of Magnetic Cataclysmic Variables," The Astrophysical Journal, Vol. 622, No. 1, 2005, p. 589. doi:10.1086/427914

[26] K. Mukai and P. A. Charles, "Orbital Motion of the Secondary in Three AM HER Systems-Evidence for LowMass White Dwarfs," Monthly Notices of the Royal Astronomical Society, Vol. 226, 1987, p. 209.

[27] T. Shahbaz and J. H. Wood, "The System Parameters of the Polars MR SER and ST LMi," Monthly Notices of the Royal Astronomical Society, Vol. 282, No. 2 1996, p. 362.

[28] T. E. Harrison, H. L. Osborne and S. B. Howell, "The Detection of Abundance Anomalies in the Infrared Spectra of Cataclysmic Variables: Shorter Period Systems," The Astronomical Journal, Vol. 129, No. 5, 2005, p. 2400.

[29] P. Szkody, J. Liebert and R. J. Panek, "IUE Results on the AM Herculis Stars CW 1103, E1114, and PG 1550," Astrophysical Journal, Vol. 293, 1985, pp. 321-327. doi:10.1086/163239

[30] Szkody, Paula., Mateo, Mario., Downes, Ronald. 1988, PASP, 100, 362S

[31] R. González-Riestra, A. Cassatella and W. Wamsteker, 
"White Dwarfs in Cataclysmic Variables - Low State IUE Observations of V794 Aquilae, MR Serpentis, and an Ursae Majoris," Astronomical Society of the Pacific Publications, Vol. 100, 1988, p. 362.

[32] P. M. Rodríguez-Pascual, R. N. González-RiestraSchartel and W. Wamsteker, "The IUE INES System: Improved Data Extraction Procedures for IUE," Astronomy and Astrophysics Supplement, Vol. 139, 1999, p. 183.

[33] M. Livio and J. E. Pringle, "Star Spots and the Period Gap in Cataclysmic Variables," Astrophysical Journal, Vol. 427, 1994, pp. 956-960. doi:10.1086/174202

[34] K. Wu and L. L. Kiss, "High and Low States of the System AM Herculis," Astronomy and Astrophysics, Vol. 481, No. 2, 2008, p. 433.
[35] A. Boggess, F. A. Carr, D. C. Evans, D. Fischel, H. R. Freeman, C. F. Fuechsel, D. A. Klingle Smith, V. L. Krueger, G. W. Longanecker and J. V. Moore, "The IUE Spacecraft and Instrumentation," Nature, Vol. 275, No. 5679, 1978, pp. 372-377. doi:10.1038/275372a0

[36] S. R. Rosen, K. O. Mason and F. A. Cordova, "PhaseResolved Optical Spectroscopy of the AM HER System E1405-451," Monthly Notices of the Royal Astronomical Society, Vol. 224, 1987, p. 987.

[37] F. Bruhweiler, "Private Communication," 2002.

[38] D. D. Lenz and T. R. Ayers, "Errors Associated with Fitting Gaussian Profiles to Noisy Emission-Line Spectra," Publications of the Astronomical Society of the Pacific, Vol. 104, 1992, p. 1104. 